\title{
Opportunities in the Cross-Scale Collaborative Human Sensing of 'Developing' Device-Free and Wearable Systems
}

\author{
Shijia Pan \\ span24@ucmerced.edu \\ University of California Merced \\ Merced, CA
}

\begin{abstract}
This is a position paper that discusses the challenges of emerging new sensing modalities for both device-free and wearable sensing systems, as well as opportunities lying in the combination of them across multiple information scales. With the development of the Internet of Things (IoT), many devices with sensing-ability have entered people's life. These systems mainly fall into two categories: wearables and infrastructure sensing (device-free). In this paper, we first briefly summarize the state-of-the-art sensing modalities of these two categories, then we discuss the challenges faced by them. We envision a future of IoT human sensing systems that achieves seamless sensing across multiple scales through collaborative information inference by both categories of modalities. Finally, we discuss the opportunities to expand the boundaries of sensing modalities that lie in their collaborative adaptation.
\end{abstract}

\section{CCS CONCEPTS}

- Human-centered computing $\rightarrow$ Ambient intelligence; Mobile devices.

\section{KEYWORDS}

Device-free sensing, wearable sensing, collaborative sensing

\section{ACM Reference Format:}

Shijia Pan and Phuc Nguyen. 2020. Opportunities in the Cross-Scale Collaborative Human Sensing of 'Developing' Device-Free and Wearable Systems . In The 2nd ACM Workshop on Device-Free Human Sensing (DFHS'20), November 15, 2020, Virtual Event, Japan. ACM, New York, NY, USA, 6 pages. https://doi.org/10.1145/3427772.3429394

\section{INTRODUCTION}

With the development of the Internet of Things (IoT), many devices with sensing-ability entered people's life. According to Statista [1], the connected devices are projected to reach 21.5 billion units worldwide by 2025. The research on sensor networks dated back to the 70s focused on three key aspects: sensing, communication, and computing [2]. With the development in these aspects, many humancentered smart applications are enabled by reliable communication

Permission to make digital or hard copies of all or part of this work for personal or classroom use is granted without fee provided that copies are not made or distributed for profit or commercial advantage and that copies bear this notice and the full citation on the first page. Copyrights for components of this work owned by others than ACM must be honored. Abstracting with credit is permitted. To copy otherwise, or republish, to post on servers or to redistribute to lists, requires prior specific permission and/or a fee. Request permissions from permissions@acm.org.

DFHS'20, November 15, 2020, Virtual Event, Japan

(c) 2020 Association for Computing Machinery.

ACM ISBN 978-1-4503-8192-5/20/11 . .\$15.00

https://doi.org/10.1145/3427772.3429394

\author{
Phuc Nguyen \\ vp.nguyen@uta.edu \\ University of Texas at Arlington \\ Arlington, TX
}

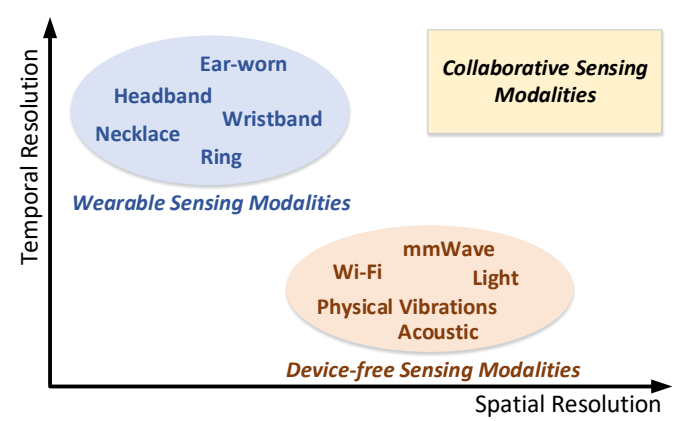

Figure 1: Collaborative wearable and device-free sensing.

over different range scales, the significant increase in computational power and decrease in size, various new sensing modalities that are less intrusive. Especially in recent years, the evolution of newly developed sensing modalities falls into two categories: on-body (wearable, mobile) and device-free (infrastructure) sensing. They have been designed to capture information in different scales including and not limited to physical and physiological scales.

Given the broad conversation around on-body (wearable) and infrastructure (device-free) human sensing, let us begin by defining the scope of this paper and some of the terms used. In this paper, we focus on the innovation of non-obtrusive 'developing' sensing technologies in IoT systems. We refer to device-free sensing as a wide range of static or semi-static sensors deployed onto infrastructure and do not move during their data acquisition - homes, offices, cars, shops, schools, airplanes, spaceship, and more. These device-free sensors typically have the ability to unobtrusively sense human physical activities including presence, breathing, heart-rate, walking, talking, running, and so on. We refer to an on-body sensor as a wearable device to monitor human physiological and emotional stages including emotion, sleeping, driving, eating, health condition, and so on. On-body sensors carried by a human can come and go from the environment over short or long-time scales.

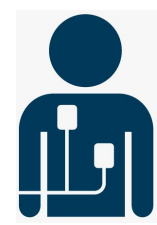

Physiological scale

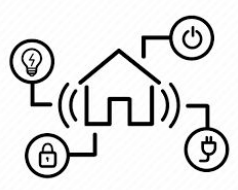

Action/Context scale

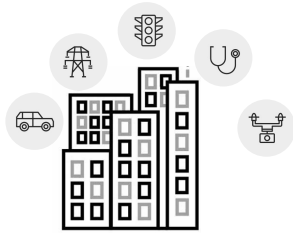

Group/Area scale
Figure 2: Multi-scale IoT sensing systems face different spatiotemporal requirements. 
Device-free human sensing is often applied in the long-term monitoring where the devices often require a reliable power supply, or in the scenario where the target user may not suitable for wearing/carrying devices on-body. For example, in-home older adults' daily care, or long-term patient physical condition monitoring is typical to target applications for device-free human sensing systems. The challenges faced by device-free human sensing are mainly from the complexity of the deployment environment, especially for various newly explored indirect information inference approaches. For example, structural vibration-based human sensing is significantly impacted by the structural characteristic itself [3]. On the other hand, the newly developing wearables can capture high-resolution information, e.g., limb movement, muscle movement, EEG, EOG, EMG, heart-rate variability, blood oxygen signals, in a non-obtrusive way. The key challenges in wearable sensing are the extremely low-amplitude, frequency-overlapped, locationdependent, user-dependent signals captured by existing low-energy budget sensing technology. As an example, while human brain signal, muscle signal, and eye movement signal are important for enabling advanced health-care monitoring, human-computer interaction applications, they are often at $u V / m V$ level and dominated by human motion artifacts.

We see opportunities in the complementary characteristics of device-free and wearable sensors (Fig. 1). We present potential research directions that allow device-free sensing to support wearable sensing and vice versa. In particular, although the device-free and wearable-based human sensing systems often have different spatiotemporal resolutions, they demonstrate complementary characteristics and cross-scale (Fig. 2) information acquisition ability. We also discuss sensing density and coverage problems in devicefree sensing and describe how wearable devices can potentially help. Last but not least, we discuss how the co-located device-free and wearable sensors can collaboratively achieve high-quality data acquisition and fine-grained information inference.

\section{STATE-OF-THE-ART SENSING MODALITIES}

In this Section, we briefly summarize the state-of-the-art sensing modalities of both device-free and wearable approaches.

\subsection{Device-free human sensing}

Various device-free human sensing modalities have been explored, such as vision $[4,5]$, acoustic [6, 7], vibration [3, 8-11], light $[12,13]$, WiFi [14, 15], mmWave [16-19], RFID [20, 21], etc. These sensing modalities acquire human information without requiring the target human subject to wear or to carry any devices. These state-of-theart sensing modalities have been used to acquire the information include but not limited to identity $[9,17]$, location $[3,22]$, activity $[10,18]$, physical status such as heart rate variability [16, 23], sleep stages [24], gait parameters [11], etc. We summarized their advantages and disadvantages in Table 1, and these characteristics determined their target or suitable applications.

\subsection{Wearable-based human sensing}

On-body sensors that are used to monitor brain signal (EEG), muscle signal (EMG), eye signal (EOG), heart-rate variability (HRV), GSR (galvanic skin response), blood pressure, breathing behaviors, activities, and so on have tremendous value in inferring the user's health, mental, physiological, and physical states. The key advantage of wearable devices is their direct contact with human skin allowing them to capture extremely low-amplitude signals from the human body. For example, using just facial muscle signals (i.e., electromyography (EMG)) alone, one can infer the stress level of a user $[25,26]$ and the eating habit and the type of food consumed by the user [27-29]. When combining these EMG signals with brain signals (i.e., electroencephalogram (EEG)), one can further understand the user's emotional states[30,31], his pain [32] and suffering level [33], or sedation level during surgery [34]. The key disadvantage of wearable sensors is its low spatial resolution. Due to its limited knowledge about the environmental condition, the device may require re-calibration every time the experiment is conducted at a new location.

\section{CHALLENGES}

IoT sensing systems have been designed targeting applications of different scales as shown in Figure 2. These applications have different spatiotemporal requirement and challenges.

\section{1 (C1) Challenges for sensing data from 'developing' modalities}

The major challenges for these two categories of sensing data are twofold 1) low signal to noise ratio (SNR) due to the indirect inference nature of the modalities, 2) various data distribution changes from human and environment.

3.1.1 (C1.1) Low SNR. For indirect device-free sensing modalities, they are often considered to be more acceptable in terms of the perceived privacy of the users compared to 'developed' modalities such as computer vision. Due to their indirect inference natural, these systems are often prone to ambient noises and hence have limited ability to acquire high-resolution information (e.g., physiological level). Furthermore, because of this, these systems' installation and maintenance are sensitive to the ambient environments and hardware configuration, which makes the procedure labor intensive. While recent scholarly publications as well as emerging wearable companies such as Emotiv [35], NeuroSky MindWave [36], BrainLink Pro [37], Muse [38], Kokoon [39], Versus [40], Neuroon Open [41], Naptime [42], etc. hold great hopes for the future of wearable sensing, there are still many challenges to be overcome. For example, heavy noises created by motion and coupled from the

Table 1: Device-free sensing modalities comparison.

\begin{tabular}{||lcc||}
\hline Modality & Pros & Cons \\
\hline \hline vision & $\begin{array}{c}\text { accurate } \\
\text { high resolution }\end{array}$ & $\begin{array}{c}\text { need light-of-sight (LoS) } \\
\text { privacy concerns }\end{array}$ \\
\hline acoustic & $\begin{array}{c}\text { accurate } \\
\text { does not require LoS }\end{array}$ & $\begin{array}{c}\text { prone to ambient noise } \\
\text { privacy concerns }\end{array}$ \\
\hline light & non-intrusive & limited resolution \\
\hline vibration & $\begin{array}{c}\text { non-intrusive } \\
\text { does not require LoS }\end{array}$ & $\begin{array}{c}\text { sensitive to structure } \\
\text { limited resolution }\end{array}$ \\
\hline mmWave & $\begin{array}{c}\text { high resolution } \\
\text { does not require LoS }\end{array}$ & $\begin{array}{c}\text { impacted by multi-path } \\
\text { impacted by metal }\end{array}$ \\
\hline WiFi & $\begin{array}{c}\text { non-intrusive } \\
\text { does not require LoS }\end{array}$ & $\begin{array}{c}\text { sensitive to } \\
\text { environment change }\end{array}$ \\
\hline
\end{tabular}


environment in daily use is the long-standing challenge limiting the practical uses of wearable bio-signal sensing systems, as it is difficult to ensure high fidelity signals.

3.1.2 (C1.1) High variation. Since the indirect device-free sensing modalities infer the human information from their interaction with the environment, the data distribution could significantly change over different environment [3] and person's physiological conditions [9]. As for wearables, the signals collected by wearables are often weak and frequency-overlapped. For example, human physiological signals are often at $u V / m V$ level and have overlapping frequency - brain signal (EEG: 1-15 Hz), muscle signal (EMG: $\geq 50 \mathrm{~Hz}$ ), eye movement signals (EOG: $0-50 \mathrm{~Hz}$ ). Signals captured from different users are different due to the dynamic of the human biological and physiological structure [43]. Designing universal solutions that work across environments and users is, therefore, extremely challenging.

\section{2 (C2) Spatiotemporal coverage v.s. resolution}

There is a trade-off between the spatiotemporal coverage and resolution for both categories of the systems. Since device-free sensing systems are often static or semi-static and have limited sensing range - e.g. at a room level - they can only capture human information while the target is in the range. This indicates a high spatial resolution but limited temporal coverage in terms of sensing. Such temporal limitation doesn't exist in wearable sensing since the devices are carried by a person from place to place. This ensures a high temporal coverage but a low spatial resolution as the wearables signal does not reflect the location characteristics. ${ }^{1}$

Indeed, we anticipate that new wearable devices and device-free sensors need to be suited for the hardware, software limitations of each system, to be connected to reliable cloud services, to be configured to collaboratively exchange information among one another. For example, distributed device-free sensing information - those associated with a specific wearer - can be aggregated to build a reliable, large-scale, and real-time infrastructure sensing map. However, realizing that idea is difficult due to the following challenges: (1) there is no reliable architecture network protocol that connects wearable and device-free sensors at a large-scale (e.g., city-level); (2) wearable devices have limited computational resource and power, designing a reliable solution to connect them to a large number of device-free sensors is challenging; (3) the overheads and benefits of utilizing wearable devices to enhance device-free sensing temporal coverage and utilizing device free sensing to enhance wearables spatial resolution are little known.

\section{3 (C3) Dataset of 'developing' new modalities}

The 'developing' sensing modalities is often limited by datasets, especially for those indirect sensing modalities with data of high distribution variation and low signal strength. For device-free sensing, taking structural vibration-based human sensing as an example, the systems utilize structures as sensors to indirectly infer human information and are sensitive to the structural characteristics at the deployment. As a result, for pure data-driven approaches, it

\footnotetext{
${ }^{1}$ Note that for mobile system that tries to optimize the area exploration and coverage is out of the scope of this paper.
}

indicates the requirement of labeled data for each deployed system/environment, which makes it impractical for large scale deployment and/or system maintenance. For wearables, human variation, including how an individual wears the device, as well as individual behavior/motion difference would cause the data distribution over different users to change. As a result, for pure data-driven approaches, it indicates labeled data collection for each individual user, which makes the technology difficult to be applied for a large scale user group. These challenges are less of a problem for 'developed' sensing modalities such as computer vision datasets [44] or IMU-based human activity recognition (HAR) datasets [45]. In recent years, efforts have been made for these 'developing' sensing modalities dataset sharing $[46,47]$. However, due to the lack of standardized data acquisition procedures and hardware, the lack of datasets of these new sensing modalities is still a challenge before we can make these systems more pervasive and adaptive.

\section{4 (C4) System cost and data quality}

The trade-off between lowing the system cost and enhancing the sensing data quality is challenging for IoT sensing systems. For person- and room-scale systems, the high cost would make the system difficult to be affordable by the individual or family. For city and area-scale systems, high-cost devices make it difficult to achieve high density or large coverage of the deployment. The state-of-the-art approaches including utilizing the physics model to enhance the data-driven estimation with limited high-resolution sensors [48], utilizing the mobility of the platform to enhance the coverage of the system with limited devices $[49,50]$. However, the challenges remain. To further systematically enhance the sensing system data quality, the environmental and hardware impacts on the data needs to be quantified by key impact factors.

\section{POTENTIAL RESEARCH DIRECTIONS}

We identify a few of the key research questions in terms of developing a reliable and cross-scale collaborative device-free and wearable-based human sensing systems.

\section{1 (D1) Enhance information acquisition resolution through collaborative sensing}

The device-free and wearable-based human sensing systems often demonstrate complementary characteristics because they have different speciotemporal resolutions and coverage. As a result, one direction or opportunities is to combine device-free and wearablebased sensing to enhance the human information acquisition's spatiotemporal resolution.

4.1.1 (D1.1) Temporal knowledge transfer for device-free systems via wearable. On-body sensors involve the deployment of extremely reliable and high-resolution sensing sensors to monitor human physical stages - emotion, eating/drinking habits, stress, pain, oxygen level, blood pressure - resulting in smart wearables. On the other hand, device-free human sensing systems at home, cars, shops, schools, and more to capture lower-resolution user activities - walking, talking, running, breathing, etc. In a collaborative sensing scenario, temporal knowledge obtained from high-fidelity sensing data obtained by wearable sensors can be used to improve/enhance device-free sensing capability. Specifically, wearable sensors can 
be used to characterize the background noises at different environments for physics-informed transfer learning for device-free systems. Moreover, wearable sensing information can be exploited to train device-free systems to enable new sensing capabilities. As an example, clean EMG, EEG, EOG signals captured by wearable sensors can be used to train vision-based sensing systems to enable human emotion monitoring (happiness, sadness, surprise, fear, anger, disgust, and contempt monitoring) using device-free sensors. In addition, monitoring human activities in the multi-person scenarios such as family gatherings, students in a class, people in a meeting, passengers on a train/car/airplane is challenging with existing device-free sensors. In these scenarios, wearable devices can provide its identity to surrounding device-free sensors allowing these infrastructure sensors to be able to identify patterns and extract signals from individuals.

4.1.2 (D1.2) Spatial knowledge enhancement for wearable via devicefree systems. The performance of wearable sensors is heavily suffered by ambient factors, especially human artifact, movement, and mobility (C2). Existing physiological sensing techniques only perform reliably with 'static' or 'semi-static' environments such as (1) sleep [43], (2) sitting [32, 51, 52], driving [53], building wearable systems that still work during everyday activities is challenging. In a collaborative sensing environment, the wearable devices can exploit sensing information provided by device-free systems to remove these noises. In particular, device-free systems can monitor environmental noises (e.g., magnetic field, electric field, acoustic, .etc.) and transfer that knowledge to wearable devices for selfconfiguration. In addition, the ability to localize user location at centimeter-level using an existing device-free system such as lightbased [54], Wi-Fi-based [55], mmWave-based [56], and ultrasoundbased [57] techniques would greatly benefit wearable devices in multi-person sensing scenario.

\section{2 (D2) Information inequality between device-free and wearable human sensing}

Due to the nature of these systems (device-free v.s. wearable), the sensing ability of them is different when applied in different scales of IoT sensing systems. For example, for human sleep monitoring, the wearable can be designed to acquire EEG, EOG, and EMG [43], while device-free sensing can capture motion or action level of information $[24,58]$. Wearables can easily capture the personscale information with finer granularity compared to device-free approaches. For room-scale and city-scale information acquisition (e.g., occupant localization or traffic pattern prediction), infrastructure sensing (device-free) $[59,60]$ would provide spatial information that allows accurate inference, which the wearable is often not designed to capture. Instead of considering this inequality as a constraint (C4), we can utilize such information inequality over multi-scale IoT systems to enable cross-scale cross-modality information association. Once such association is established, the well 'developed' knowledge in one can be transferred to the 'developing' knowledge learning of the other. For example, we consider the sleep stages and their relation to EEG, EOG, and EMG signals are well 'developed' knowledge, which can be transferred to the co-located structural vibration-based sleep monitoring system. This would allow the establishment of the relation between in-sleep motion and sleep stage to monitor users who do not or cannot wear wearable.

\section{3 (D3) Collaboratively reduce deployment density and configuration requirement}

One of the common research goals for IoT sensing systems is to reduce the system cost, which includes but is not limited to the price of the device, the number of devices needed to ensure coverage, power consumption, installation \& maintenance effort $(\mathrm{C} 1$, C4). Wearables enable continuous monitoring, which can provide feedback on maintaining device-free deployment efficiency with minimum devices. On the other hand, the environmental or context information provided by device-free systems could enable wearable sensing with fewer devices, which makes the wearable deployment more practical for everyday usage. Besides, given the dynamic of sensor availability at any time, designing a reliable solution to authenticate data sources and users, are critical. Collaborative design can associate biometrics, which can be easily measured by wearables, and in-situ context, which can be acquired from device-free human sensing, to enable effort free data sources authentication.

\section{4 (D4) Quantify quality of sensing as a service}

Up to this point, we have covered different approaches of combining the two types of sensors mostly at the research and development stage - building prototypes, we now discuss how their sensing information can be leveraged to asset each other performance during a deployment. We define the quality of sensing (QoSen) as a relation between measurable cyber (hardware) and physical (environmental) factors and the system performance (e.g., classification accuracy). For device-free human sensing systems, various environmental factors could impact the QoSen, including and not limited to ambient noise level, signal propagation path and medium [61]. The hardware configuration could also impact the acquired signal (after ADC), including analog filter, amplifier gain, ADC resolution, etc. [62]. For wearables, environmental factors impacting the QoSen include and are not limited to amplitude over noise level (e.g., the electrical, magnetic field, acoustic noises). Hardware configuration could impact the ability to extract overlapped frequency signals, including analog/digital filter, notch filter, amplifier, ADC architecture. In particular, designing proper filters and $\mathrm{ADC}$ architecture (i.e., > 24 bits, $\Sigma-\Delta$ architecture) is a must for most physiological wearable sensing devices. To quantify these impact factors through the sensing signals enables the system self-diagnose on the quality of the sensing procedure, which can be used to further 'debug' the IoT sensing system deployment and enhance the system performance. The complementary information from device-free and wearable sensing systems would enable automatic assessment for this quality of sensing by leveraging the shared-context of co-located systems.

\section{SUMMARY}

In this paper, we discussed the opportunities to push the boundaries of both device-free and wearable-based sensing modalities based on their collaborative adaptation. The future of IoT human sensing poses great opportunities for collaborative device-free and wearable sensing at homes, cars, offices, schools, airplanes, and beyond. There are many challenges in developing reliable and cross-scale device-free and wearable-based human sensing systems. Exploiting collaborative wearable and device-free sensing would significantly improve both categories of systems' performances as well as enable new exciting applications. 


\section{REFERENCES}

[1] Internet of things - active connections worldwide 2015-2025. https://www.statista $\mathrm{com} /$ statistics/1101442/iot-number-of-connected-devices-worldwide/. Accessed 2020-09-07.

[2] Chee-Yee Chong and Srikanta P Kumar. Sensor networks: evolution, opportunities, and challenges. Proceedings of the IEEE, 91(8):1247-1256, 2003.

[3] Mostafa Mirshekari, Jonathon Fagert, Shijia Pan, Pei Zhang, and Hae Young Noh. Step-level occupant detection across different structures through footstepinduced floor vibration using model transfer. Fournal of Engineering Mechanics, 146(3):04019137, 2020

[4] Zhe Cao, Gines Hidalgo, Tomas Simon, Shih-En Wei, and Yaser Sheikh. Openpose realtime multi-person $2 \mathrm{~d}$ pose estimation using part affinity fields. arXiv preprint arXiv:1812.08008, 2018

[5] Riza Alp Güler, Natalia Neverova, and Iasonas Kokkinos. Densepose: Dense human pose estimation in the wild. In Proceedings of the IEEE Conference on Computer Vision and Pattern Recognition, pages 7297-7306, 2018

[6] Zheng Sun, Aveek Purohit, Kathleen Yang, Neha Pattan, Dan Siewiorek, I Lane, and P Zhang. Coughloc: Location-aware indoor acoustic sensing for non-intrusive cough detection. In International Workshop on Emerging Mobile Sensing Technologies, Systems, and Applications. Citeseer, 2011.

[7] Wei Xu, ZhiWen Yu, Zhu Wang, Bin Guo, and Qi Han. Acousticid: gait-based human identification using acoustic signal. Proceedings of the ACM on Interactive, Mobile, Wearable and Ubiquitous Technologies, 3(3):1-25, 2019.

[8] Shijia Pan, Amelie Bonde, Jie Jing, Lin Zhang, Pei Zhang, and Hae Young Noh Boes: building occupancy estimation system using sparse ambient vibration monitoring. In Sensors and Smart Structures Technologies for Civil, Mechanical, and Aerospace Systems 2014, volume 9061, page 90611O. International Society for Optics and Photonics, 2014.

[9] Shijia Pan, Tong Yu, Mostafa Mirshekari, Jonathon Fagert, Amelie Bonde, Ole J Mengshoel, Hae Young Noh, and Pei Zhang. Footprintid: Indoor pedestrian identification through ambient structural vibration sensing. Proceedings of the ACM on Interactive, Mobile, Wearable and Ubiquitous Technologies, 1(3):1-31, 2017.

[10] Amelie Bonde, Shijia Pan, Mostafa Mirshekari, Carlos Ruiz, Hae Young Noh, and Pei Zhang. Oac: Overlapping office activity classification through iot-sensed structural vibration. In Proceedings of the 5th International Conference on Internet of Things Design and Implementation, 2020.

[11] Jonathon Fagert, Mostafa Mirshekari, Shijia Pan, Pei Zhang, and Hae Young Noh Characterizing structural changes to estimate walking gait balance. In Dynamics of Civil Structures, Volume 2, pages 333-335. Springer, 2019.

[12] Tianxing Li, Chuankai An, Zhao Tian, Andrew T Campbell, and Xia Zhou. Human sensing using visible light communication. In Proceedings of the 21st Annual International Conference on Mobile Computing and Networking, pages 331-344, 2015.

[13] Tianxing Li, Qiang Liu, and Xia Zhou. Practical human sensing in the light. In Proceedings of the 14th Annual International Conference on Mobile Systems, Applications, and Services, pages 71-84, 2016.

[14] Fadel Adib, Zach Kabelac, Dina Katabi, and Robert C Miller. 3d tracking via body radio reflections. In 11th \{USENIX\} Symposium on Networked Systems Design and Implementation (\{NSDI\} 14), pages 317-329, 2014.

[15] Fadel Adib, Zachary Kabelac, and Dina Katabi. Multi-person motion tracking via rf body reflections. 2014

[16] Eyhab Al-Masri and Misba Momin. Detecting heart rate variability using millimeter-wave radar technology. In 2018 IEEE International Conference on Big Data (Big Data), pages 5282-5284. IEEE, 2018.

[17] Peijun Zhao, Chris Xiaoxuan Lu, Jianan Wang, Changhao Chen, Wei Wang Niki Trigoni, and Andrew Markham. mid: Tracking and identifying people with millimeter wave radar. In 2019 15th International Conference on Distributed Computing in Sensor Systems (DCOSS), pages 33-40. IEEE, 2019.

[18] Chris Xiaoxuan Lu, Muhamad Risqi U Saputra, Peijun Zhao, Yasin Almalioglu, Pedro PB de Gusmao, Changhao Chen, Ke Sun, Niki Trigoni, and Andrew Markham. milliego: mmwave aided egomotion estimation with deep sensor fusion. arXiv preprint arXiv:2006.02266, 2020.

[19] Peijun Zhao, Chris Xiaoxuan Lu, Bing Wang, Changhao Chen, Linhai Xie, Mengyu Wang, Niki Trigoni, and Andrew Markham. Heart rate sensing with a robot mounted mmwave radar. In 2020 IEEE International Conference on Robotics and Automation (ICRA), pages 2812-2818. IEEE, 2020.

[20] Daqiang Zhang, Jingyu Zhou, Minyi Guo, Jiannong Cao, and Tianbao Li. Tasa: Tag-free activity sensing using rfid tag arrays. IEEE Transactions on Parallel and Distributed Systems, 22(4):558-570, 2010.

[21] Xuanke He, Jiang Zhu, Wenjing Su, and Manos M Tentzeris. Rfid based noncontact human activity detection exploiting cross polarization. IEEE Access, 8:46585-46595, 2020.

[22] Chenren Xu, Bernhard Firner, Robert S Moore, Yanyong Zhang, Wade Trappe, Richard Howard, Feixiong Zhang, and Ning An. Scpl: Indoor device-free multisubject counting and localization using radio signal strength. In Proceedings of the 12th international conference on Information processing in sensor networks, pages 79-90, 2013.
[23] Zhenhua Jia, Musaab Alaziz, Xiang Chi, Richard E Howard, Yanyong Zhang, Pei Zhang, Wade Trappe, Anand Sivasubramaniam, and Ning An. Hb-phone: a bedmounted geophone-based heartbeat monitoring system. In 2016 15th ACM/IEEE International Conference on Information Processing in Sensor Networks (IPSN), pages 1-12. IEEE, 2016.

[24] Zhizhang Hu, Emre Sezgin, Simon Lin, Pei Zhang, Hae Young Noh, and Shijia Pan. Device-free sleep stage recognition through bed frame vibration sensing. In Proceedings of the 1st ACM International Workshop on Device-Free Human Sensing, pages 39-43, 2019.

[25] C-M Tsai, S-L Chou, Elliot N Gale, and Willard D McCall. Human masticatory muscle activity and jaw position under experimental stress. fournal of oral rehabilitation, 29(1):44-51, 2002.

[26] Ulf Lundberg, Roland Kadefors, Bo Melin, Gunnar Palmerud, Peter Hassmén, Margareta Engström, and Ingela Elfsberg Dohns. Psychophysiological stress and emg activity of the trapezius muscle. International journal of behavioral medicine, 1(4):354-370, 1994.

[27] K Kohyama, L Mioche, and P Bourdio3. Influence of age and dental status on chewing behaviour studied by emg recordings during consumption of various food samples. Gerodontology, 20(1):15-23, 2003.

[28] Laurence Mioche, Pierre Bourdiol, Jean-Francois Martin, and Yolande Noël. Variations in human masseter and temporalis muscle activity related to food texture during free and side-imposed mastication. Archives of Oral Biology, 44(12):10051012,1999

[29] Kaoru Kohyama, Laurence Mioche, and Martin Jean-Francois. Chewing patterns of various texture foods studied by electromyography in young and elderly populations. Fournal of Texture Studies, 33(4):269-283, 2002.

[30] Xiao-Wei Wang, Dan Nie, and Bao-Liang Lu. Emotional state classification from eeg data using machine learning approach. Neurocomputing, 129:94-106, 2014.

[31] Klaus-Robert Müller, Michael Tangermann, Guido Dornhege, Matthias Krauledat, Gabriel Curio, and Benjamin Blankertz. Machine learning for real-time singletrial eeg-analysis: from brain-computer interfacing to mental state monitoring. fournal of neuroscience methods, 167(1):82-90, 2008.

[32] Hoang Truong et al. Painometry: wearable and objective quantification system for acute postoperative pain. In Proceedings of the 18th International Conference on Mobile Systems, Applications, and Services, MobiSys '20, pages 419-433. Association for Computing Machinery.

[33] J Sarnthein, A Morel, A Von Stein, and D Jeanmonod. Thalamic theta field potentials and eeg: high thalamocortical coherence in patients with neurogenic pain, epilepsy and movement disorders. Thalamus \& Related Systems, 2(3):231238, 2003.

[34] C De Deyne, Michel Struys, Johan Decruyenaere, J Creupelandt, E Hoste, and Francis Colardyn. Use of continuous bispectral eeg monitoring to assess depth of sedation in icu patients. Intensive care medicine, 24(12):1294-1298, 1998.

[35] Emotiv brainwear. https://goo.gl/dLrJBV. [Online; accessed Sept. 24, 2020].

[36] NeuroSky MindWave. https://goo.gl/cEf7fi. [Online; accessed Sept. 24, 2020].

[37] BrainLink Pro. https://goo.gl/hrxuAb. [Online; accessed Sept. 24, 2020].

[38] Muse. https://goo.gl/5zwtcJ. [Online; accessed Sept. 24, 2020].

[39] Kokoon. https://goo.gl/tr3QJV. [Online; accessed Sept. 24, 2020].

[40] Versus. https://goo.gl/ubW3T7. [Online; accessed Sept. 24, 2020].

[41] Neuroon Open. https://goo.gl/5WyTuj. [Online; accessed Sept. 24, 2020]

[42] Naptime. https://goo.gl/iY3Ber. [Online; accessed Sept. 24, 2020].

[43] Anh Nguyen, Raghda Alqurashi, Zohreh Raghebi, Farnoush Banaei-kashani, Ann C. Halbower, and Tam Vu. A lightweight and inexpensive in-ear sensing system for automatic whole-night sleep stage monitoring. In Proceedings of the 14th ACM Conference on Embedded Network Sensor Systems CD-ROM, SenSys '16, pages 230-244, 2016.

[44] Jia Deng, Wei Dong, Richard Socher, Li-Jia Li, Kai Li, and Li Fei-Fei. Imagenet: A large-scale hierarchical image database. In 2009 IEEE conference on computer vision and pattern recognition, pages 248-255. Ieee, 2009.

[45] Ricardo Chavarriaga, Hesam Sagha, Alberto Calatroni, Sundara Tejaswi Digumarti, Gerhard Tröster, José del R Millán, and Daniel Roggen. The opportunity challenge: A benchmark database for on-body sensor-based activity recognition. Pattern Recognition Letters, 34(15):2033-2042, 2013.

[46] DATA'18: Proceedings of the First Workshop on Data Acquisition To Analysis, New York, NY, USA, 2018. Association for Computing Machinery.

[47] DATA'19: Proceedings of the 2nd Workshop on Data Acquisition To Analysis, New York, NY, USA, 2019. Association for Computing Machinery.

[48] Xinlei Chen, Xiangxiang Xu, Xinyu Liu, Shijia Pan, Jiayou He, Hae Young Noh, Lin Zhang, and Pei Zhang. Pga: Physics guided and adaptive approach for mobile fine-grained air pollution estimation. In Proceedings of the 2018 ACM International foint Conference and 2018 International Symposium on Pervasive and Ubiquitous Computing and Wearable Computers, pages 1321-1330, 2018.

[49] Jingxiao Liu, Bingqing Chen, Siheng Chen, Mario Bergés, Jacobo Bielak, and HaeYoung Noh. Damage-sensitive and domain-invariant feature extraction for vehicle-vibration-based bridge health monitoring. In ICASSP 2020-2020 IEEE International Conference on Acoustics, Speech and Signal Processing (ICASSP), pages 3007-3011. IEEE, 2020. 
[50] Xinlei Chen, Susu Xu, Jun Han, Haohao Fu, Xidong Pi, Carlee Joe-Wong, Yong Li, Lin Zhang, Hae Young Noh, and Pei Zhang. Pas: Prediction-based actuation system for city-scale ridesharing vehicular mobile crowdsensing. IEEE Internet of Things fournal, 7(5):3719-3734, 2020.

[51] Phuc Nguyen, Nam Bui, Anh Nguyen, Hoang Truong, Abhijit Suresh, Matt Whitlock, Duy Pham, Thang Dinh, and Tam Vu. TYTH-Typing On Your Teeth Tongue-Teeth Localization for Human-Computer Interface. In Proceedings of the 16th Annual International Conference on Mobile Systems, Applications, and Services, pages 269-282. ACM, 2018.

[52] Nam Bui et al. eBP: A wearable system for frequent and comfortable blood pressure monitoring from user's ear. In The International Conference on Mobile Computing and Networking, MobiCom '19, pages 1-17. ACM.

[53] Nhat Pham et al. Wake: A behind-the-ear wearable system for microsleep detection. In Proceedings of the 18th International Conference on Mobile Systems, Applications, and Services, MobiSys '20, page 404-418, New York, NY, USA, 2020. ACM.

[54] Vikram Iyer, Elyas Bayati, Rajalakshmi Nandakumar, Arka Majumdar, and Shyamnath Gollakota. Charging a smartphone across a room using lasers. 1(4):143:1143:21.

[55] Deepak Vasisht, Swarun Kumar, and Dina Katabi. Decimeter-level localization with a single WiFi access point. pages $165-178$.

[56] Joan Palacios, Guillermo Bielsa, Paolo Casari, and Joerg Widmer. Singleand multiple-access point indoor localization for millimeter-wave networks.
18(3):1927-1942. Conference Name: IEEE Transactions on Wireless Communications.

[57] An ultrasonic based indoor positioning system - IEEE conference publication.

[58] Mingmin Zhao, Shichao Yue, Dina Katabi, Tommi S Jaakkola, and Matt T Bianchi. Learning sleep stages from radio signals: A conditional adversarial architecture. In International Conference on Machine Learning, pages 4100-4109, 2017.

[59] Mau-Tsuen Yang, Rang-Kai Jhang, and Jia-Sheng Hou. Traffic flow estimation and vehicle-type classification using vision-based spatial-temporal profile analysis. IET Computer Vision, 7(5):394-404, 2013.

[60] Susu Xu, Lin Zhang, Pei Zhang, and Hae Young Noh. An indirect traffic monitoring approach using building vibration sensing system. In Proceedings of the 14th ACM Conference on Embedded Network Sensor Systems CD-ROM, pages 374-375, 2016.

[61] Yue Zhang, Lin Zhang, Hae Young Noh, Pei Zhang, and Shijia Pan. A signal quality assessment metrics for vibration-based human sensing data acquisition. In Proceedings of the Second Workshop on Data Acquisition To Analysis. ACM, 2019.

[62] Shijia Pan, Susu Xu, Mostafa Mirshekari, Pei Zhang, and Hae Young Noh. Collaboratively adaptive vibration sensing system for high-fidelity monitoring of structural responses induced by pedestrians. Frontiers in Built Environment, 3:28, 2017. 